\title{
Effect of Lyophilization and Various Treatments on Spectral Characteristics of Beef Muscle Tissue and Its Main Components
}

\author{
Alla P. Nechiporenko*, Ul'yana Yu. Nechiporenko, Ludmila V. Plotnikova, Aleksandr V. Podshivalov, \\ Sergey V. Myakin, Mayya V. Uspenskaya
}

Research University of information technologies, mechanics and optics, E-mail: allanech2512@yandex.ru

\begin{abstract}
Native and lyophilized shredded muscle tissue of beef and its main ingredients (muscle fibers, stroma, stroma proteins) prepared via consecutive treatment with water, saline (Weber solution ) and alkaline (0.6 M NaOH) solutions as well as colloidal solutions-extracts of sarcoplasmic substances, actomyosin and mucopolysaccharide complexes are comparatively studies by attenuated total internal reflection infrared (ATIR) and diffuse reflectance electronic spectroscopy. A high sensitivity, efficiency, extended options and advanced prospects of diffuse spectroscopy are considered in respect of the characterization of the component composition of complex solid-state and liquid biological systems in medical and veterinary practice.
\end{abstract}

Keywords: Muscle Tissue; Solid and Liquid Biological Systems; IR Spectroscopy; Diffuse Reflection Spectroscopy

\section{Introduction}

Infrared spectroscopy, including a modern technique of attenuated total internal reflection infrared (ATIR is an advanced approach in the study of various properties of solid and liquid biological systems ${ }^{[1,2]}$. Physical principles of vibrational spectroscopy afford the analysis of the composition of a broad range of functional groups featuring with the same IR absorption bands regardless of types of host substances. Unfortunately, the presence of water markedly affects IR spectroscopy data due to "masking" a significant part of the determined functional groups thus interfering with the analysis and interpretation of spectra relating to biological tissues usually containing a large amount of water. Although elimination of interference from water is facilitated by the lyophilization of samples, it leads to considerable changes of native chemical bonds.

Diffuse reflectance electronic spectroscopy (DRES) generally provides less advanced options, however in respect of characterization of biological objects composition in some cases it provides information inaccessible using other techniques, particularly due to the lack of interference from water absorbing radiation in the range of vacuum ultraviolet $(<200 \mathrm{~nm})$. Widely used for the characterization of inorganic materials surfaces, DRES method has not yet found a proper application in the study of materials of biological origin.

UV-spectra of organic substances are highly specific. The presence of certain bands in these spectra are generally determined by selective absorption due to * and $\mathrm{n} *$ electronic transitions in chromophore groups $(\mathrm{C}=\mathrm{O}, \mathrm{C}=\mathrm{C}, \mathrm{C} \equiv \mathrm{C}, \mathrm{N}=\mathrm{N}, \mathrm{N}=\mathrm{O}, \mathrm{C}=\mathrm{C}-\mathrm{C}=\mathrm{O}$ conjugated systems, aryl rings, oxygen in ester/ether groups) and their environment ${ }^{[3-5]}$. The data required for the identification of structural units in biomolecules (proteins, lipids, carbohydrates) relates to spectra in the medium- and near-UV regions (200-400 nm). Analytical features of applied methods, resolution and informative value depend on the discreteness of the excitation energies mostly relating to unshared electron pairs in carbonyl $(\mathrm{C}=\mathrm{O})$ and ether $(=\mathrm{O})$ groups in almost all classes of biological tissue constituents.

No reference data on DRES application for the characterization of biological tissues are available so far, except for the studies of animal muscle tissues and their solid components performed by our research group since

\footnotetext{
Copyright (C) 2018 Alla P. Nechiporenko et al.

doi: 10.24294/jacs.v1i2.494

EnPress Publisher LLC.This work is licensed under the Creative Commons Attribution-NonCommercial 4.0 International License (CC BY-NC 4.0). http://creativecommons.org/licenses/ by/4.0/
} 
$2009^{[6-9]}$. Later this approach was used by S.M.Orehova as the main research method in the study of pork muscle surface irradiated by accelerated electrons of different power ${ }^{[10-12]}$. However, the considered studies related only to was always solid-phase systems of different nature and anatomic localization ${ }^{[13-15]}$. In this context it was of interest to study liquid extracts of muscle tissue main components and compare the data of two reflection spectroscopy techniques as tools with different functionalities and operating in different spectral (energy) regions.

The aim of this work was to study optical characteristics of the surface of native shredded beef muscle tissues and their main components (muscle fiber, stroma, and stroma proteins), determined using different techniques widely applied in academic and scientific laboratories as well as in food industry ${ }^{[16-18]}$, based on the difference and a hierarchical sequence in their solubility resulting from various chemical treatments. Another objects of studies were liquid fractions yielded during such consecutive treatments, i.e. extracts of sarcoplasmic substances, actomyosin and mucopolysaccharide complexes.

\section{Experimental}

The objects of study involved chilled muscle tissue of beef (longissimus of the back), subjected to the following 4-stage preparation for consecutive separation of the main components (including spectrophotometric control of the completeness of chemical treatment at the stages 2-4):

1 - double grinding of the muscle tissue through $2.5 \mathrm{~mm}$ circular dies;

2 - muscle fiber preparation by repeated extraction of sarcoplasmic (water-soluble portion containing all types of the present compounds) of minced muscle tissue with distilled water until clear water;

3 - stroma (connective tissue consisting of a protein component and a carbohydrate) preparation by removal of actomyosin complex (contractile proteins - actin, myosin, tropomyosin) from the muscle fibers with saline Weber $(\mathrm{NaCl} 10 \%)$ followed by subsequent laundering of the extractant distilled water;

4 - stromal proteins collagen and elastin (connective tissue proteins) separation by extraction of mucopolysaccharides (carbohydrate structural component of connective tissue) with $0.6 \mathrm{M} \mathrm{NaOH}$ solution in the cold followed by washing of the solvent with distilled water.

The first aqueous, saline and alkaline colloid solutions were prepared by centrifugation for the study of optical performances of the extracted components of sarcoplasma, actomyosin and mucopolysaccharide complexes, respectively.

After each processing stage, samples of the basic parts of muscular tissue and extracts were analyzed by attenuated total internal reflection IR-spectroscopy (ATIR) and diffuse reflection electron spectroscopy (DRES). Vibrational spectra were recorded using Fourier-spectrometer Tensor 37 with a diamond ATR element (Bruker, Germany) and software package OPUS in the frequency range $4000-600 \mathrm{~cm}^{-1}$ in the acquisition mode. DRES spectra were measured using Specord M-200 (AIZ Engineering GmbH, Germany) spectrophotometer in the wavelength range 200-700 nm in the absorption mode. Spectra of liquid extracts were measured using PTFE cell liner with the reflection coefficient close to that of standard Spectrolon reference sample. Furthermore, to increase the differentiability of the spectra tissue samples and extracts were subjected to lyophilization after each stage of processing by freeze-drying using Triad 7400030, (Labconco, USA) equipment for $48-72$ hours at temperature $-30^{\circ} \mathrm{C}$ and vacuum pressure of $165 \mathrm{PA}$.

\section{Results and discussion}

ATIR data. Figure 1 shows IR spectra of samples obtained as a result of successive aqueous, saline and alkaline treatments of beef muscle. The observed intensive high frequency bands at $3450 \mathrm{~cm}^{-1}$ can relate to valent oscillations of $\mathrm{NH}_{\mathrm{n}}$ - and $\mathrm{OH}$-groups all insoluble and water-soluble components of sarcoplasm, muscle fibers, stroma, stroma proteins, as well as hydroxyl groups and associated water molecules. A moderate intensity band at $1637 \mathrm{~cm}^{-1}$ corresponds to deformation vibrations of $\mathrm{OH}$-groups of water in all the samples ${ }^{[19]}$. 


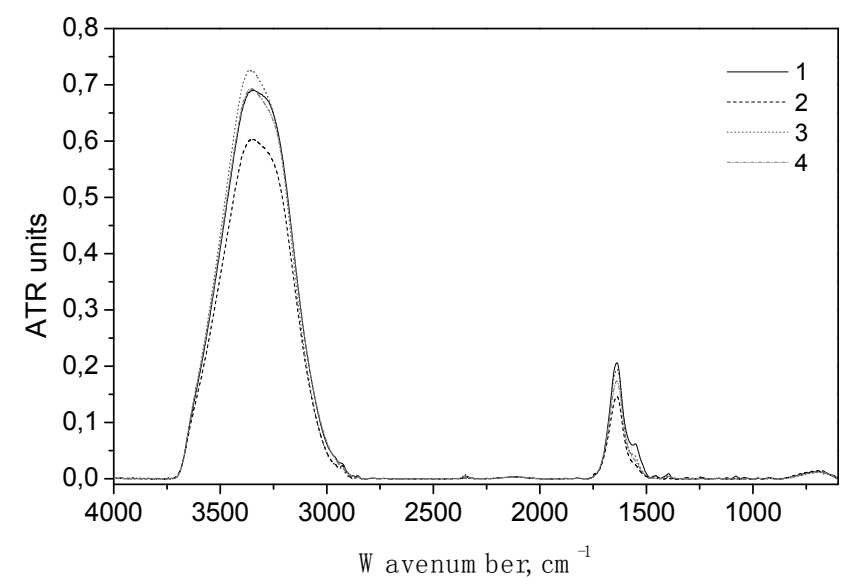

Figure 1; - IR spectra of the original minced muscle tissue (1) beef and her. Main components: 2 - muscle fiber; 3 stroma; 4 - stroma proteins.

At the right shoulder of this band in the spectra of muscle tissue and muscle fibers weak bands relating to carboxylate carbonyls $(\mathrm{C}=\mathrm{O})$ of Amide II is observed at $1560 \mathrm{~cm}^{-1}$. In the range below $1500 \mathrm{~cm}^{-1}$ deformation vibrations of $\mathrm{CH}_{\mathrm{n}}-, \mathrm{NH}_{\mathrm{n}}-\mathrm{OH}$-groups and stretching vibrations of the bonds $\mathrm{C}-\mathrm{O}, \mathrm{C}-\mathrm{C}, \mathrm{C}-\mathrm{N}$ etc. can occur. However, water shields the peaks of most functional groups. Extraction of water-soluble components of sarcoplasm (stage 2) leads to a decrease in the intensity of the complex band at $3450 \mathrm{~cm}^{-1}$ and bands corresponding to deformation vibrations of associated OH-groups in water molecules. On the contrary, salt extraction of actomyosin protein complex (stage 3) and alkaline - mucopolysaccharide component of the stroma (stage 4) cause an increase in intensity and small high frequency shift of the bands at $3450 \mathrm{~cm}^{-1}$. The spectrum of stroma proteins is similar to that of distilled water due to their high water absorption.

Lyophilization of muscle tissue and its components significantly leads to significant changes in spectral characteristics of their surface generally reflecting the processes taking place during the consecutive extractions.

IR spectra of lyophilized samples of muscle tissue after each stage of processing (Figures 2 and $\mathbf{3}$ ) indicate that in all cases dehydration (moisture removal) leads to the appearance and differentiation of the main intrinsic absorption bands. After extraction of sarcoplasm (stage 2 ), in addition to carbohydrate and lipid components containing a number of water-soluble globular proteins, a decrease in the intensity of most bands, including the bands of stretching vibrations of carbonyls $(\mathrm{C}=\mathrm{O})$ Amide I (1638-1640 $\left.\mathrm{cm}^{-1}\right)$ and Amide II (1529-1534 $\left.\mathrm{cm}^{-1}\right)$ is observed, except for the bands corresponding to vibrations of $\mathrm{CH}_{\mathrm{n}^{-}}\left(3060-2851 \mathrm{~cm}^{-1}\right)$ and $-\mathrm{COOH}(1744$ $\mathrm{cm}^{-1}$ ) functional groups in lipid (fatty acids), protein (amino acids) and mucopolysaccharide structural units on the surface of muscle fibers.

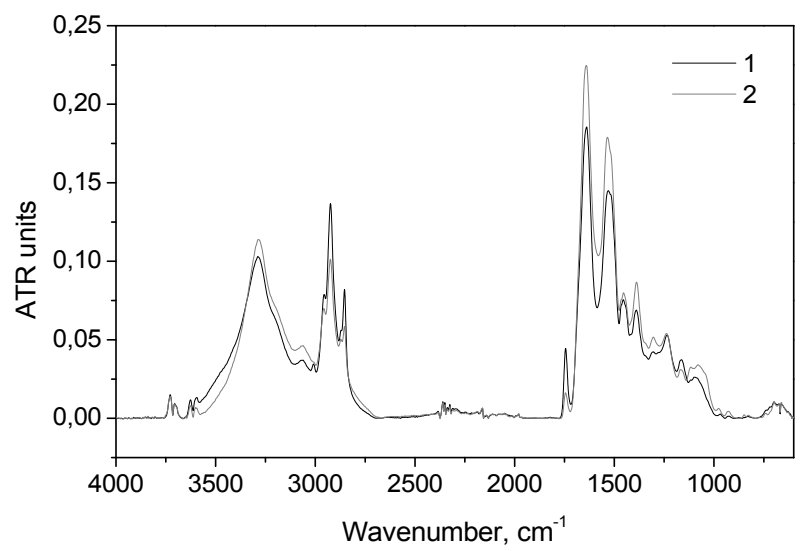

Figure 2; - IR spectra of lyophilized muscle tissue (1) and muscle fibers (2).

Salt processing of the muscle fibers (stage 3), results in the decrease of spectrum bands intensity almost over the whole measured frequency range with a small high frequency shift of the absorption bands of carbonyl groups of Amide II from $1529 \mathrm{~cm}^{-1}$ to $1559 \mathrm{~cm}^{-1}$ (Figure 3) due to the extraction of protein actomyosin complexes. On the contrary, the removal of polysaccharide component (partially shielding the bands relating to functional groups of connective tissue proteins) from the stroma with alkaline extractant (stage 4), leads to the increase in intensity of all major bands intrinsic to the overall spectrum of collagen and elastin proteins.

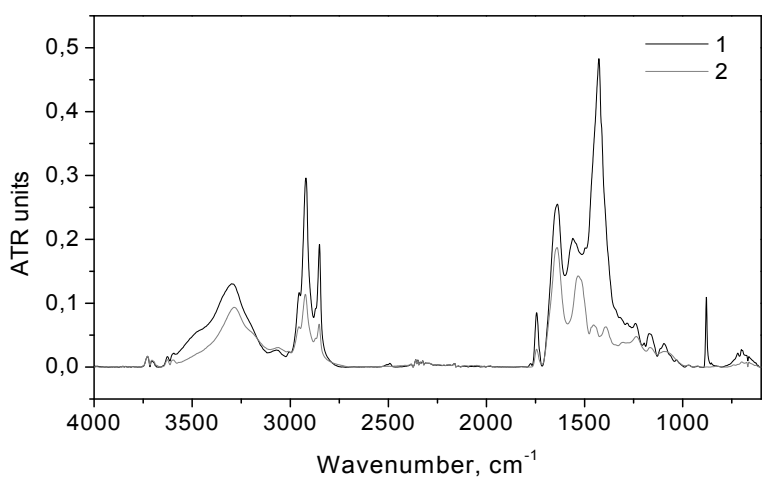

Figure 3; - IR spectra of lyophilized stroma (1) and stroma proteins (2). 
The absorption spectrum (2) features with a narrow band of medium intensity at $879,30 \mathrm{~cm}^{-1}$ present only in the spectrum of stroma proteins and likely relating to amino acid Hydroxyproline predominant in the collagen composition. Furthermore, a prominent intensive strip $1427 \mathrm{~cm}^{-1}$ is observed featuring with a certain broadening probably due to the deformation vibrations of different types of $-\mathrm{CH}_{\mathrm{n}}$-groups. A series of weak bands in the region $1390-1000 \mathrm{~cm}^{-1}$ present in the spectra of all samples can be associated with vibrations of $\mathrm{N}-\mathrm{H}, \mathrm{C}-\mathrm{N}, \mathrm{C}-\mathrm{O}$ and $\mathrm{C}-\mathrm{O}-\mathrm{C}$ linkages ${ }^{[19]}$. The increased water absorption ability of collagen is indicated by broadening of the band at $3229 \mathrm{~cm}^{-1}$ corresponding to asymmetric vibrations of NH-groups, with the appearance of its left "shoulder" at about 3500 $\mathrm{cm}^{-1}$.

DRES data. DRES spectra of the chopped freshly chilled beef muscle and its main components are presented in Figure 4.

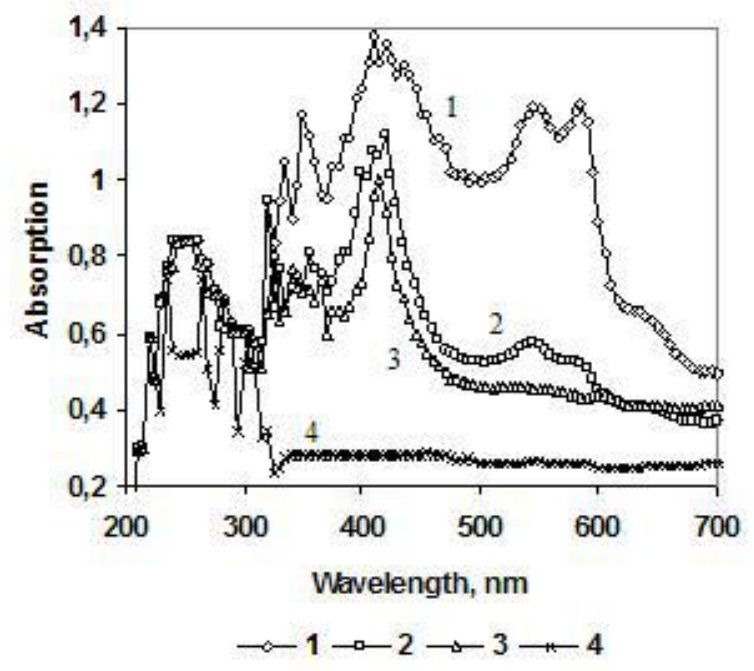

Figure 4; - DRES spectra of the original minced beef muscle (1), muscle fibers (2), stroma (3) and stroma proteins (4).

The electronic spectrum of animal muscular tissue surface is represented by four quite different regions. In the visible range, double band relating to pigmented protein myoglobin $(545 / 585 \mathrm{~nm})$ and another band corresponding to its oxidized form of methemoglobin $(635 \mathrm{~nm})$ responsible for a gray-brown hue of meat are observed. Mucopolysaccharides provide an intensive band at 400-425 nm, probably including a band intrinsic to Sora due to the absorption of Gemma myoglobin. A series of bands of medium intensity in the
near-UV region $(320-380 \mathrm{~nm})$ relate to the building blocks of the lipid component, i.e. unsaturated fatty acids (oleic, linoleic, linolenic and arachidonic).

The absorption in the middle UV region (240-300 $\mathrm{nm}$ ) is determined by chromophores of four amino acid residues (tyrosine, tryptophan, phenylalanine, histidine $\left.{ }^{[5]}\right)$, disulfide bond $(230 / 235 \mathrm{~nm})$, as well as monosaccharides, oligosaccharides and their various hybrids (270-315 nm). The peptide bond gives a band at 220-225 nm. However, despite this versatility, the building blocks of substances of the same class, regardless of the nature of the constituent parts of muscle tissue, absorb in a relatively narrow range of wavelengths. The overall decline across the spectrum, its fragment or separate band of differentiation of bands indicate the removal of the corresponding substances or their destruction under the effect of certain external or internal factors.

The extraction of sarcoplasm leads to leaching of water soluble substances of all types, resulting in reduced light absorption of the muscle fibers (spectrum 2). However the double band relating to myoglobin remains and disappears only after salt treatment (spectrum 3) providing the removal of actomyosin fibrils associated with myoglobin. The bands corresponding to lipid components (320-380 nm) and mucopolysaccharides $(415-420 \mathrm{~nm})$ remain after saline processing are disappear after alkaline treatment of stroma in the cold. Thus, the spectrum (5) in UV-region corresponds to stroma. It should be noted that the peaks at 220, 235, 365, $285 \mathrm{~nm}$ correspond to the appearance of protein structures, while minimums at 275, 295 and $315 \mathrm{~nm}$ relate to carbohydrates.

Lyophilisation of the muscle tissue samples after treatment with water, salt and alkaline solutions demonstrates the changes in optical characteristics of the samples surface by removing moisture during the consecutive extraction of the components from its ingredients. Spectra of the lyophilized samples shown in figure 5 . 


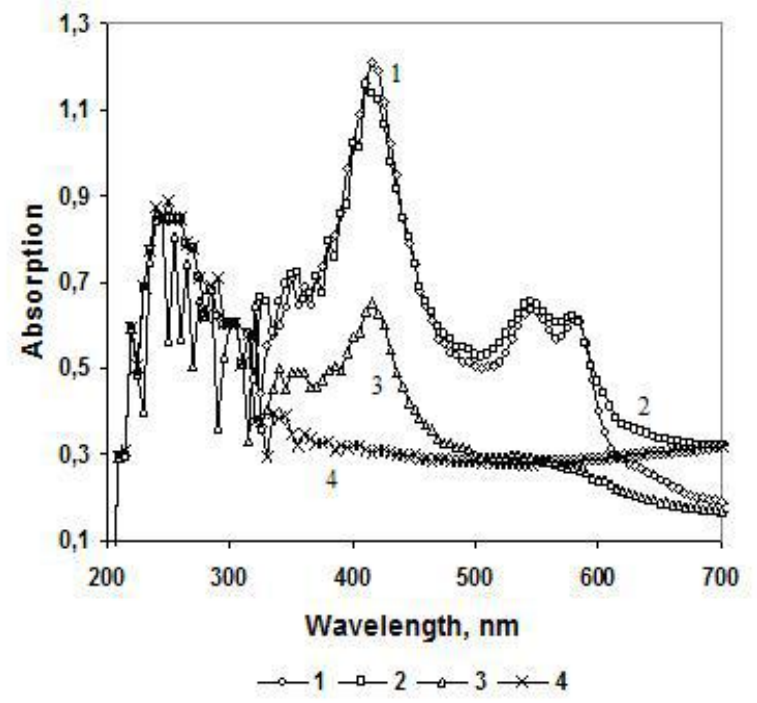

Figure 5; - DRES spectra of lyophilized samples of minced muscle tissue (1), muscle fiber (2), stroma (3) and proteins of the stroma (4) beef.

The first issue noteworthy is the convergence of the spectra corresponding to lyophilized samples of muscle tissue and muscle fibers with retaining the intensity of the double band relating to myoglobin and disappearance of the band corresponding to methemoglobin. The spectrum of muscle fibers (2) features with the intensity increase and differentiation of the bands of the lipid components $320,340,355,380 \mathrm{~nm}$, which is consistent with the observed intensity growth of the absorption bands of $\mathrm{CH}_{n}-$ and $\mathrm{COOH}$-groups in IR spectra. In stroma spectrum (3), the absorption of the lipid building blocks and polysaccharides markedly drops after lyophilization in combination with structuring of the band in the region of protein-carbohydrate complex absorption (340-315 $\mathrm{nm}$ ), indicating the disruption of chemical bonds.

One of the objectives of this study was the characterization of the component composition of all the source and lyophilized extracts. The results of this study are illustrated in Figure 6. Noteworthy, spectra of aqueous lyophilized and not lyophilized extracts are generally similar to those of lyophilized muscle tissue and muscle fibers. This suggests that the aqueous extract adequately reflects the component structure and composition of functional groups on the surface of both samples. The observed differences relate to the intensity of absorption bands corresponding to lipid and polysaccharide components.
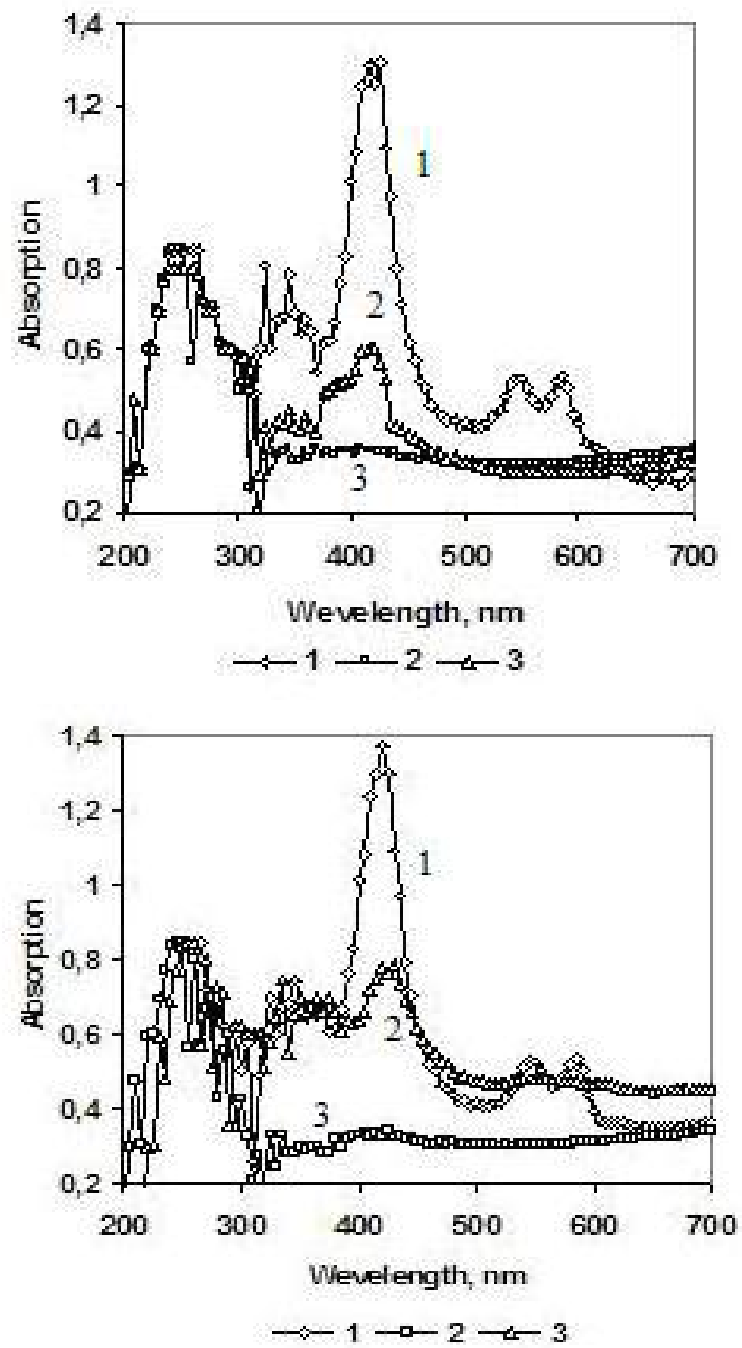

Figure 6; - DRES spectra of beef muscle aqueous (1), saline (2) and alkaline (3) extracts: a - without lyophilization, b - lyophilized.

The spectrum of saline extract indicates the predominance of protein components, as confirmed by the presence of the relating bands for lyophilized extracts. In addition, the spectrum of lyophilized extract features with weak bands correspnding to lipids and polysaccharides indicating their partial extraction with Weber solution. The spectra of alkaline extracts general reflect the stroma composition, but their lyophilisation leads to the increased intensity above $300 \mathrm{~nm}$ and bathochromic shift of the entire spectrum. However, the formation of hydrogen bonds between water and carbonyl groups is known to provide a hypsochromic shift and decrease in intensity for spectra of organic compounds upon their hydration ${ }^{[4]}$. Noteworthy, the spectra of lyophilized stroma proteins (Figure 5, 
spectrum 4) and actomyosin protein complex (Figure 6a and $6 \mathrm{~b}$, spectrum 2 ) in the middle UV region are similar to each other.

\section{Conclusion}

The performed study afforded IR and UV-vis diffused reflection spectra of the native and lyophilized surface of beef muscle tissues, their muscle fibers and stroma proteins, as well as sarcoplasm (water-soluble part of the muscle) extracts solutions, actomyosin (contractile proteins) and lipid-mucopolysaccharide complex (carbohydrate components of the stroma interspersed with lipid granules). The results obtained using two different reflection spectral techniques are found to be in good agreement. The effect of freeze-drying liophilization process upon IR and UV-vis diffuse reflection spectra of solid and liquid components of animal muscle tissue is analyzed.

The obtained results particularly illustrate the potential and prospects of DRES technique as a rapid and efficient analytical approach requiring almost no special sample preparation and diluting procedures, in respect of the study of various complex liquid biological systems (blood, plasma, serum, ejaculate, etc.). This method can be useful in medical and veterinary practice as an additional approach to initial diagnostics of various diseases and monitoring of their treatment dynamics.

\section{References}

1. Stuart BH. Infrared Spectroscopy: Fundamentals and Applications. NY. Wiley, 2004; 242 p.

2. Gordetsov A C. Infrared spectroscopy of biological fluids and tissues // Modern technology in medicine. 2010; no. 1, pp. 84 - 97.

3. Vyaz'min SY, Ryabuhin DS, Vasilev AV. Electronic spectroscopy of organic compounds. St. Petersburg, SPbLTA Publ. 2011; 43 p.

4. Sverdlova OV. Electronic spectra in organic chemistry. Leningrad: Khimiya, 1985; 248 p.

5. Demchenko A.P. Ultraviolet spectrophotometry and protein structure. K i e v: Naukova dumka Publ. 1981; 208 p.

6. Nechiporenko AP, Kuzmina MC, Tsvetkova MN. The influence of microbial processes on the acid-base surface characteristics of a bird muscle tissue // Proceedings of The Institute of Refrigeration and Biotechnology. 2007; No. 3. P. 8-13.

7. Nechiporenko AP, Shershneva KS, Nechiporenko $\mathrm{UYu}$, et al. Effect of fineness of ground beef on the acidity of its surface. // Proceedings of The Institute of Refrigeration and Biotechnology. 2008; No. 3. P 20-24.

8. Nechiporenko AP, Koroleva AA, Tsvetkova MN The effect of different types of effects on surface properties of pork meat // Proceedings of The Institute of Refrigeration and Biotechnology. 2008; No. 2. P. 25-30.

9. Nechiporenko AP, Chestnova IS, Tsvetkova MN. The influence of low temperatures on acid-base surface characteristics of minced lamb // Proceedings of The Institute of Refrigeration and Biotechnology. 2008; No. 2. P. 21-24.

10. Orehova SM. Radiation-chemical preserving muscle pork: dis. kand. tech. of Sciences, St. Petersburg: University ITMO, 2014; 175 p

11. Orehova S, Nechiporenko U, Vasileva I, et al. Electronic spectrum of pork and beef muscle tissue surface samples, subjected to electronic-irradiation processing // 6th Baltic Conference on Food Science and Technology "Innovations for food science and production". "Foodbalt 2011". Latvia, Jelgava, May 5-6. 2011; P. 199-203.

12. Orehova SM, Nechiporenko UYu, Vasil'eva IV, et al. The effect of ethanol on the microflora, acidity and electron spectrum of the surface irradiated muscle tissue of pork and beef // Proceedings of the XV all-Russian conference "Fundamental research and innovation in national research universities". St. Petersburg: Polytechnical University Publ. 2011; (2): P. 103 - 105.

13. Plotnikova LV, Nechiporenko AP, Orehova SM, et al. Examination of the muscular tissue of animal origin by methods of spectroscopy of reflection // Optics and spectroscopy. 2017; (122):No 6. P. 1051-1054.

14. Nechiporenko AP, Uspenskaya MV, Orehova SM, et al. The reflectance spectroscopy in the study of muscle tissue of animal origin. Part I // Scientific journal ITMO, 2017; 2(32): pp. 29-39.

15. Nechiporenko AP. Donor-acceptor properties of surfaces of solid-phase systems. Indicator method. St. Petersburg: Publishing house "Lan", 2017; 284 p.

16. Bazarnova YG, Burova TE, Marchenko VI, et al. Biochemical bases of the processing and storage of raw materials of animal origin. Textbook. St. Petersburg, Prospekt nauki Publ., 2011;192 p.

17. Antipova LV, Glotova IA, Rogov IA. Research methods of meat and meat products. Moscow, Kolos Publ., 2001; 376 p.

18. Sokolov AA. Physico-chemical and biochemical bases of meat products technology. Moscow: Pishchevaya promyshlennost' Publ. 1965; $489 \mathrm{p}$.

19. Tarasevich BN. The IR spectra of the main classes of organic compounds. Reference materials. Moscow, MGU Publ. 2012; 55 p. 